\title{
Cerebro-afferent vessel and pupillary basal diameter variation induced by stomatognathic trigeminal proprioception: a case report
}

\author{
Vincenzo De Cicco
}

\begin{abstract}
Introduction: A patient affected by asymmetric hemodynamics of cerebro-afferent vessels underwent duplex color scanner investigations in occlusal proprioceptive un- and rebalance conditions. Pupillometric video-oculographic examinations were performed in order to spot connected trigeminal proprioceptive motor patterns able to interfere on sympathetic autonomic activity. The aim of this case report is to verify if involuntary jaw closing during swallowing, executed in unbalance and rebalance myoelectric activity, would be able to modify cerebral hemodynamics.
\end{abstract}

Case presentation: A 56-year-old Caucasian Italian woman affected by asymmetric blood flow of cerebro-afferent vessels underwent an electromyographic investigation of her occlusal muscles in order to assess their occlusal functional balance. The extreme asymmetry of myoelectric activity in dental occlusion evidenced by electromyographic values suggested the rebalancing of the functions of occlusal muscles through concurrent transcutaneous stimulation of the trigeminal nerve supra- and submandibular motor branches. The above-mentioned method allowed the detection of a symmetric craniomandibular muscular relation that can be kept constant through the use of a cusp bite modeled on the inferior dental arch: called orthotic-syntropic bite for its peculiar use of electrostimulation. A few days later, the patient underwent a duplex color scanner investigation and pupillometric video-oculographic examinations in occlusal unbalance and rebalance conditions.

Conclusions: A comparative data analysis showed that an unbalanced dental occlusal function may represent an interferential pattern on cerebral hemodynamics velocity and pupillometric evaluations have proved useful both in the analysis of locus coeruleus functional modalities and as a diagnostic tool in the assessment of pathologies involving locus coeruleus and autonomic systems. The inclusion of myoelectric masseter examinations can be useful in patients with asymmetric hemodynamics of cerebro-afferent vessels and dental occlusal proprioceptive rebalance can integrate the complex therapy of patients with increased chronic sympathetic activity.

\section{Introduction}

Recent brain mapping studies have revealed the effects of gum chewing and voluntary dental tapping and clenching on brain function. An increasing flow of evidence has indicated that the neural activity of daily chewing consistently stimulates cerebral areas with positive effects in maintaining brain functions, associated with an increase of blood flow, supporting earlier evidence that not only the areas of cerebral cortex related to movements but also the hippocampus and prefrontal

Correspondence: vincenzodecicco4@virgilio.it

Department of Oral Science, University "G. d'Annunzio", via dei Vestini 31, Chieti 66100, Italy cortex, normally associated with memory, are activated by jaw movements [1-3]. Furthermore, an epidemiological survey and cross-sectional study with subjects whose ages ranged from 50 years to 80 years demonstrated that reduced chewing ability or dysfunctional teeth might induce senile processes with decrease of cognitive function and learning effect $[4,5]$. At the same time, findings on aged rats have demonstrated the effects generated by the loss of molar teeth with reduction of spatial memory, acetylcholine release from the parietal cortex [6] and alteration of the septohippocampal cholinergic system [7]. Yamazaki K. et al. verified that the number of extracted teeth was directly proportional to

\section{Biomed Central}


the loss of spatial memory and to the reduction of trkBmessenger ribonucleic acid (mRNA) levels [8].

Although the brain mapping study has high spatial resolution, it is unsuitable for a quantitative analysis of overall changes in cerebral blood flow associated with daily jaw chewing movements. Recent findings have utilized transcranial doppler ultrasonography to measure blood flow velocity in major cerebral blood vessels because this method provides the advantages of continuous real-time recording and ease-of-use allowing evaluation of relationships between jaw chewing and cerebral circulation [3,9]. The cited studies have correlated the change of task induced in cerebral blood flow due to the working side and to myoelectric intensity of the masseter muscle in voluntary activity. Given that chewing is a movement semi-automatically controlled by the brainstem generator pattern [10], the aim of this case report is to verify if involuntary jaw closing during swallowing, executed in unbalance and rebalance myoelectric activity, would be able to modify cerebral hemodynamics. Normally, dental occlusion takes place constantly at 1 minute intervals during swallowing and these occlusions are superior in number to voluntary chewing activity.

Pupillometry has proved useful to extend the autonomic relationships between trigeminal and vascular systems [11] because basal pupil diameter variations are correlated with the autonomic nervous system [12] and locus coeruleus (LC) activity [13]. The findings of Elam et al. [14], in fact, showed that sympathetic nerve activity is parallel to LC discharges. Also, the paragigantocellularis (PGi) nucleus of the ventral medulla is an important anatomic pathway which might mediate this relationship directly. The PGi is a critical relay for the sympathoexcitatory efferents of the autonomic hypothalamic centers that subserve both vascular muscular tone and reflexive pupillary dilation [14]. It presents afferent connections with LC and the trigeminal system [15]. Moreover, the pupillometric baseline closely tracks LC tonic discharge frequency and it is influenced by noradrenergic release ratio [13]. Neuroanatomical studies performed through an anterograde and retrograde transport method have indicated that many of the regions that received dense inputs from projected LC neurons, in turn, feed back to these coerulei neurons [16], which are uniformly sensitive to a variety of non-noxious stimuli, including tactile, visual, and auditory, with a specific degree of activation stimulus $[17,18]$. The trigeminal system is strictly connected with LC which exhibit mixed cellular elements with trigeminal mesencephalic neurons $[16,19]$. Couto et al. showed through anterograde and retrograde tract-tracing with fast blue injections reciprocal connections between the trigeminal and LC systems [20]. Moreover, Panneton et al., using anterograde transneuronal transport of the herpes simplex virus (HSV-1) into the anterior ethmoidal nerve, observed LC and PGi nuclei
HSV-1-labeled [21]. At last, coerulean and peri-coerulean areas can be activated by increasing the discharge frequency of trigeminal mesencephalic neurons activated both by masseter spindle receptors due to excessive interocclusal space [22], and by periodontal receptors for increased occlusal charge [23]. In addition, the coerulean area can also be indirectly activated by the trigeminal motor nucleus. This nucleus does not have a definite nuclear delimitation but it is mixed with lateral reticular formation (LRF) parvocellular neurons [24], and it is part of the ascending reticular activating system [25]. Presumably, neuromotor hyperactivity of the mastication preferential side elicits a concomitant asymmetric brainstem stimulation of reward reticular systems [26], including diffused projection catecholaminergic systems of intermediate reticular formation nuclei (IRFn). Previous studies recorded short latency ipsilateral electrophysiological responses electrophysiological responses in LRF and IRFn after passive mandible dislocations [27]. Finally, the basal pupillometric test can be considered a non-invasive instrument of analysis apt to examine the responsivity of trigeminal proprioception in modeling both coerulean noradrenergic activity and autonomic sympathetic activity. The present case report documents basal pupillometric dynamics, with corneal topography, and blood flow velocity in carotid (C.a.) and vertebral (V.a.) arteries, performed through duplex color scanner examinations induced by involuntary jaw clenching during swallowing in unbalance and rebalance stomatognathic proprioception.

\section{Case presentation}

The subject of our study, a 56-year-old Caucasian Italian woman, presents with an occlusal open bite and a complete dental formula, with only the left superior second premolar missing, substituted by an implantoprosthetic rehabilitation (Figure 1). The patient, a medical doctor, has a normal blood pressure range, is not affected by any metabolic disease and is a non-cigarette smoker. For the evaluation of her occlusal muscle activity, a bilateral electromyography (EMG) of her masseter muscle was recorded using an evaluation system of mandibular movement (K6-I; Myotronics, Seattle, WA, USA) and Duo-trode surface $\mathrm{Ag}-\mathrm{AgCl}$ electrodes (Duo-trode; interelectrode distance: $19.5 \mathrm{~mm}$, Myotronics). EMG data were recorded at a sampling rate of $240 \mathrm{~Hz}$ and amplified at a time constant of 0.06 seconds. For the evaluation of her muscle activity, voluntary dental clenching was executed and recorded during swallowing. In accordance with the dental diagnostic protocol [28], a preliminary evaluation of the patient's myoelectric activity in dental occlusion was performed through muscle EMG in order to assess their functional balance. Registered values showed a remarkable functional asymmetry of masseter muscles, $23 \mathrm{mV}$ for her left masseter and $103 \mathrm{mV}$ for her right 


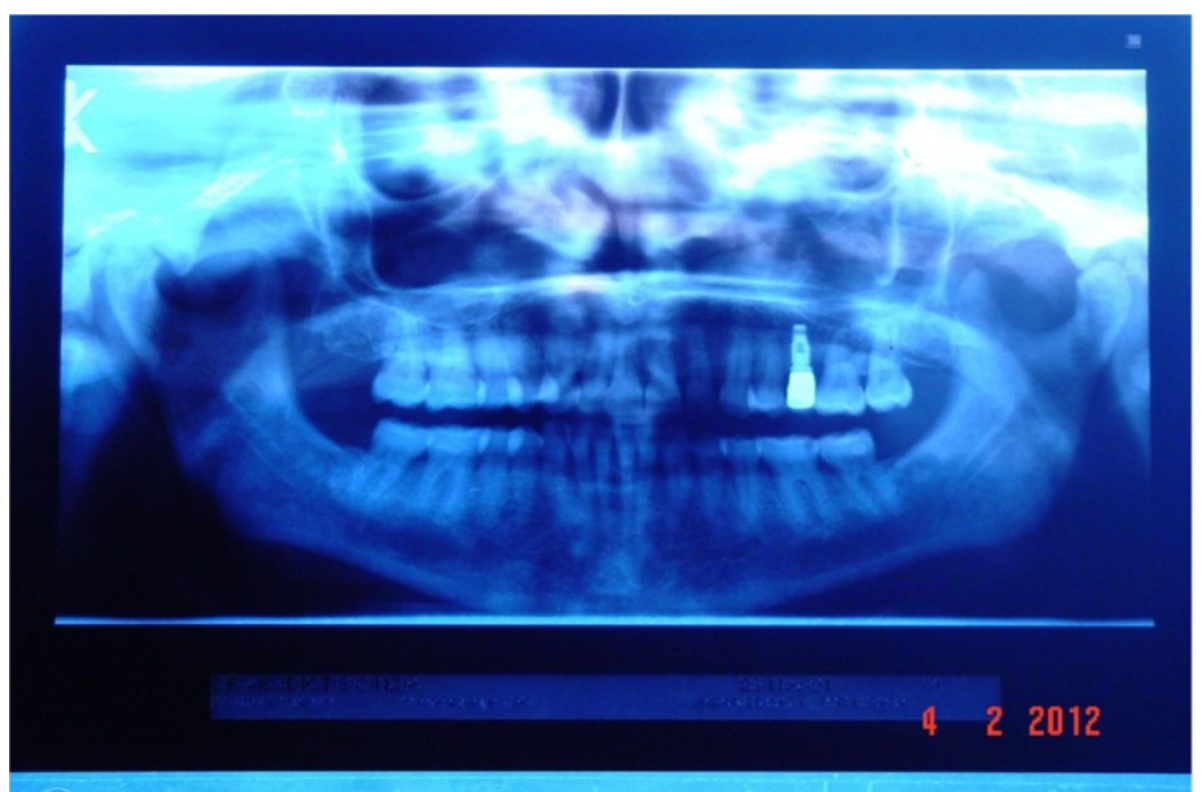

Figure 1 Orthopantomography: habitual occlusion with implant-dental prosthesis rehabilitation.

masseter (Figure 2). According to the expressed electromyographic values, muscular activity was symmetrized by applying a 15 minutes transcutaneous stimulation of trigeminal motor branches at low frequency for elevator occlusal muscles and at medium frequency for submandibular antagonist muscles. This method allowed detection of the functional trajectory of occlusal elevator muscles and to record a symmetric craniomandibular relation, positioning a self-hardening material between the dental arches. The same material was used to make a cusp bite modeled on the inferior dental arch named orthotic-syntropic bite for its peculiar use of electrostimulation. When the orthotic was applied, electromyographic control was repeated to verify occlusal myoelectric balance. Registrations have documented substantially equal values: $57 \mathrm{mV}$ for left masseter muscle and $61 \mathrm{mV}$ for right masseter (Figure 3). Immediately after, the patient was submitted to pupillometric and

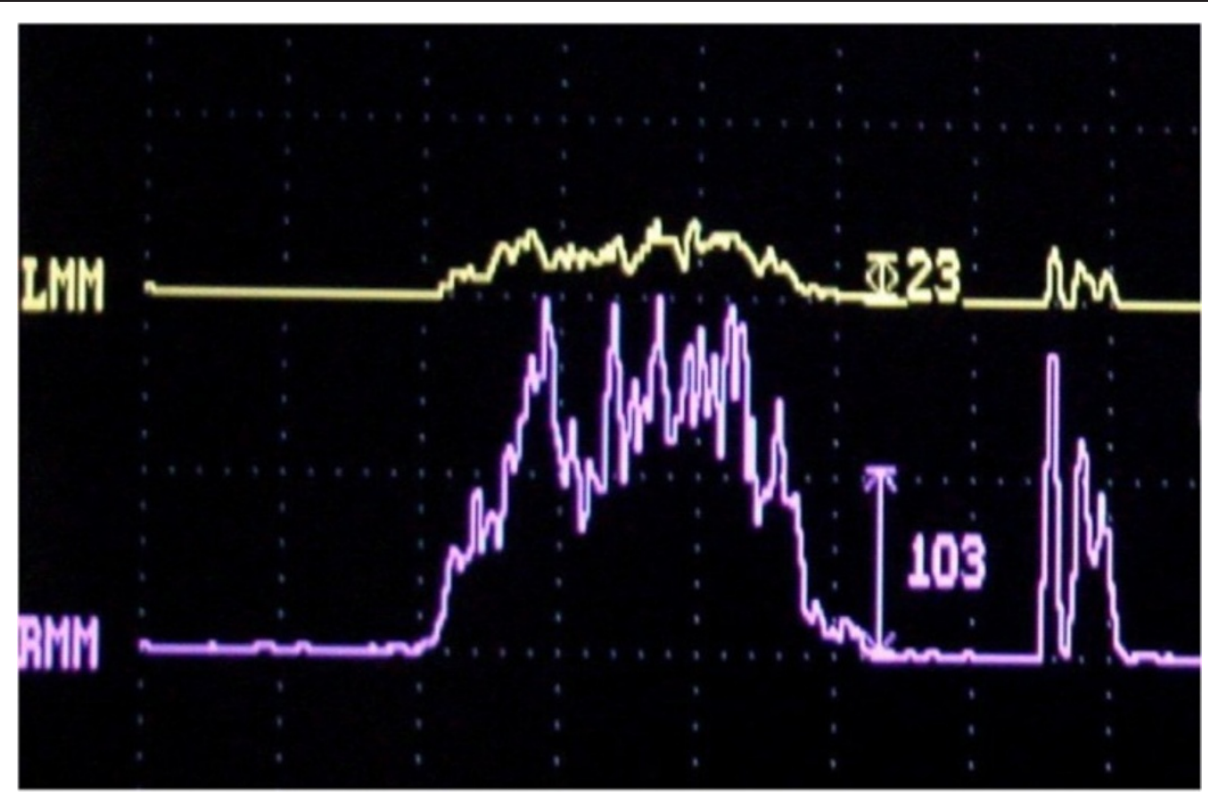

Figure 2 Electromyography values of masseters in habitual occlusion: the left myoelectric activity $23 \mathrm{mV}$, the right myoelectric activity $103 \mathrm{mV}$. 


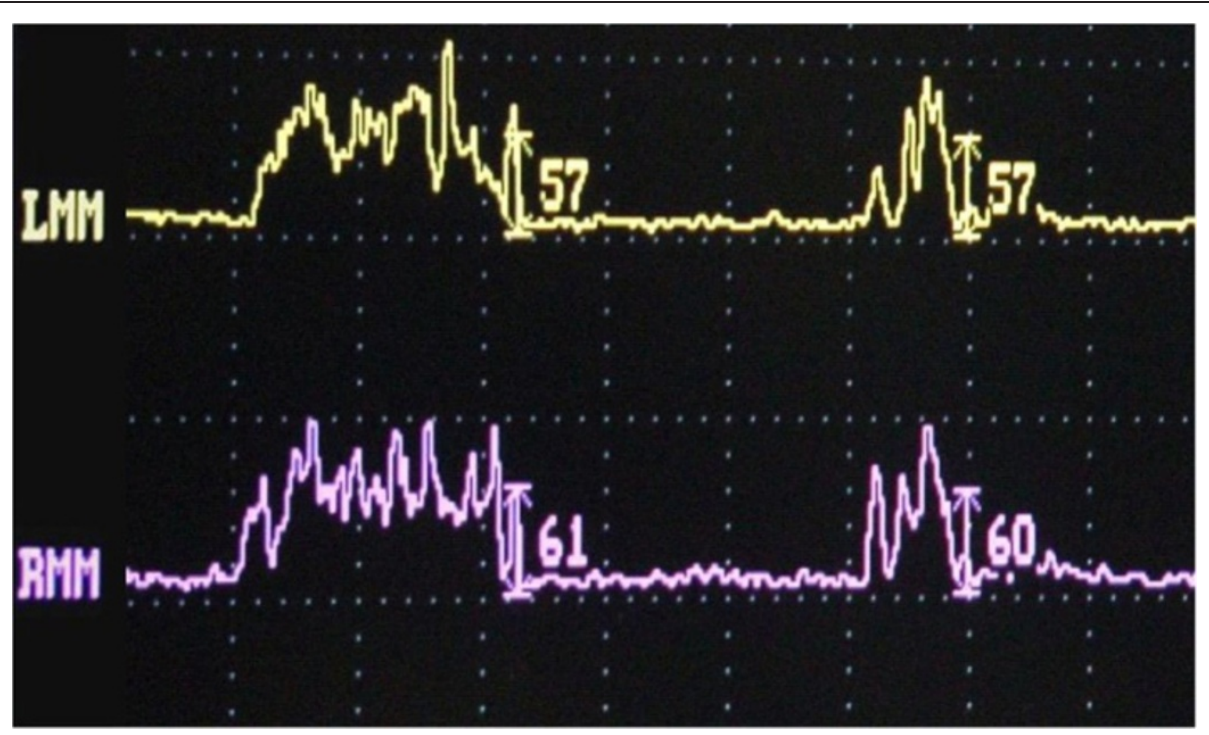

Figure 3 Electromyography values of masseters with orthotic-syntropic application: the left myoelectric activity $57 \mathrm{mV}$, the right myoelectric activity $61 \mathrm{mV}$.

hemodynamic examinations in habitual occlusion first and with the orthotic soon after.

For pupillary diameter measurement, we used a computerized corneal topographer MODI02 software 2005 LITE (CSO, Florence, Italy), made of a survey section by Placido disk 24 loops, camera sensor charge-coupled device (CCD) $1 / 3$ inch and a claim support. The instrument presents, during the pupillar acquisition phases, a constant lighting of the disk and a $56 \mathrm{~mm}$ distance of work. The points measured during data acquisition are 6.144, with a model elaboration higher than 100.000 points. Registered pupillometric analysis showed a remarkable right and left baseline asymmetry, respectively $4.98 \mathrm{~mm}$ (Figure 4) and $4.40 \mathrm{~mm}$ (Figure 5), whereas in the occlusal rebalance condition an equivalent pupil diameter was registered, $4.13 \mathrm{~mm}$ right pupil (Figure 6) and $4.10 \mathrm{~mm}$ left pupil (Figure 7). Indeed, pupillometric data analysis registered in occlusal rebalance shows a more suitable reduction of the basal diameter, with clear right side decrease, relating to higher occlusal myoelectric values.

For blood flow computerized examination, a GE HealthCare echograph, Voluson E8 Expert model, was used, with a 3D-4D-color-power Doppler volumetric probe. The duplex color scanner investigations were executed with an interval of 60 minutes, in habitual occlusion first, (Figure 8 carotid artery, Figure 9 vertebral artery) and with the orthotic after (Figure 10 carotid artery, Figure 11 vertebral artery). The following evaluations were performed (see Table 1).

systolic pulsatility and average flow velocity: (P.I. Index); systolic and diastolic relationship-flow: (R.I. Index);

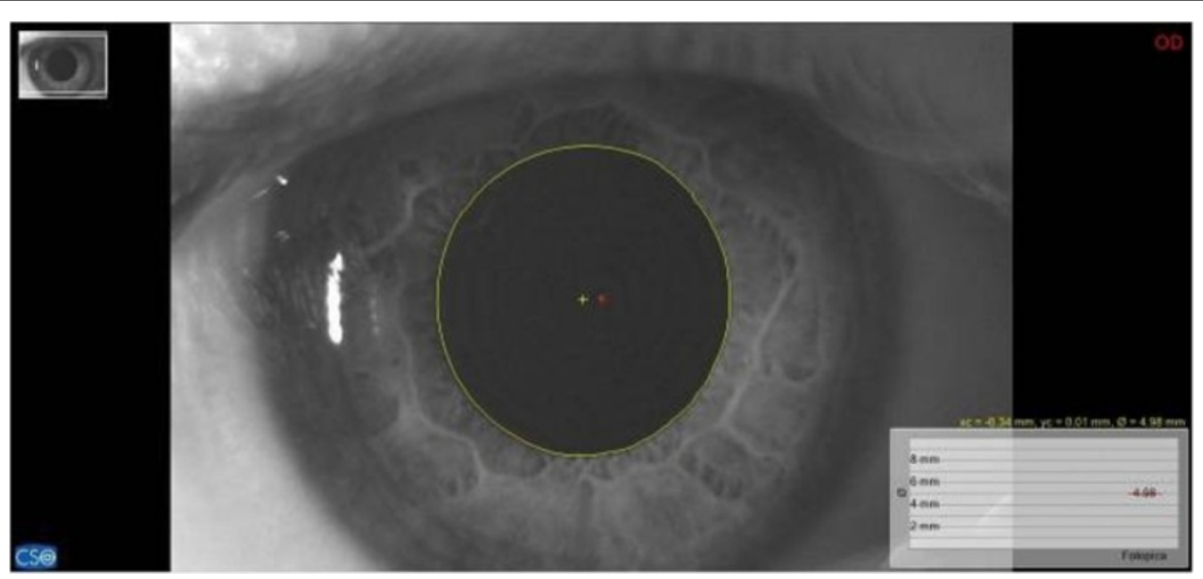

Figure 4 Basal pupillometry in habitual occlusion. Right pupil diameter: $4.98 \mathrm{~mm}$. 


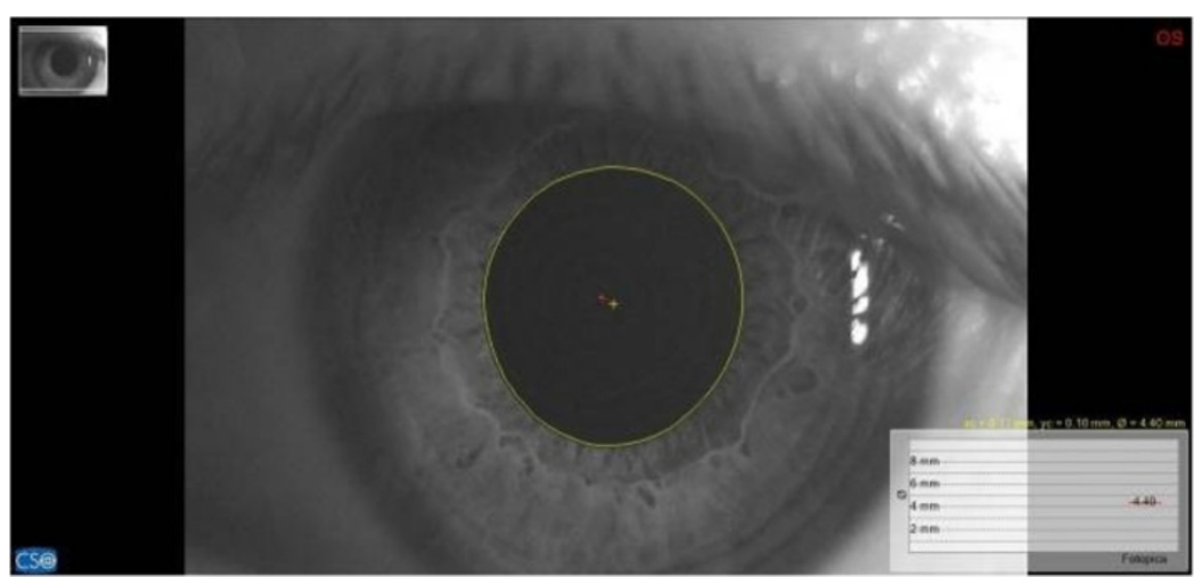

Figure 5 Basal pupillometry in habitual occlusion. Left pupil diameter: $4.40 \mathrm{~mm}$.

systolic peak in $\mathrm{cm} /$ second: (P.S. Index); diastasis cordis in $\mathrm{cm} /$ second: (E.D. Index); systole-diastole relationship: (S-D Index);

Carotid artery: C.a.;

Vertebral artery: V.a.

The registrations reveal that the patient's left V.a. hemodynamic is more influenced by trigeminal proprioception. In fact, the orthotic application reduces on the left the S-D index of 70.94 and equilibrates the values of both vertebral arteries, 3.40 (left) and 3.21 (right), respectively. Whereas, in the ED index, diastolic flow increase of $12.06 \mathrm{~cm} /$ second of the left V.a. makes the values of both arteries equal, 12.70 (left) and 12.16 (right) respectively. Moreover, in the PI index it is possible to observe that the different average flow between the right (1.0) and left (2.88) vertebral arteries is totally cancelled in occlusal rebalance, with perfectly equal values (1.23). Also the PS Index confirms the previous results because a general reduction of hemodynamic values is registered both in carotid and vertebral arteries after orthotic application. In fact, the systolic hematic peak, expressed in $\mathrm{cm} /$ second, shows decreases of 2.05 on the right and of 7.69 on the left in the carotid arteries, while in vertebral arteries the decreases are of 7.42 on the right and of 4.37 on the left. The RI index does not seem to be influenced by occlusal proprioception.

\section{Discussion}

There are reports in the literature of the effects on cerebral blood flow of voluntary chewing and of regional increases in brain neural activity, but the examinations described in the present case report show that the proprioceptive intensity during involuntary jaw occlusion in swallowing can also influence the hemodynamics in major cerebral blood vessels. The basal pupil diameter evaluations could be a valid method to connect the neurophysiologic relationships between proprioceptive trigeminal system and LC-sympathetic autonomic functional modes. The comparative analysis of the obtained

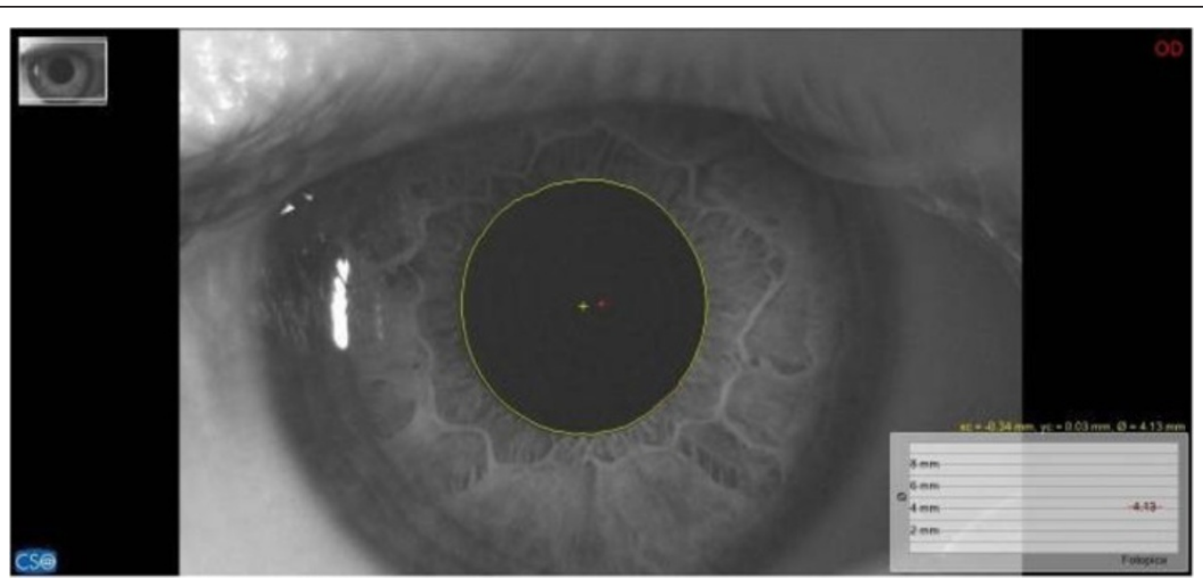

Figure 6 Basal pupillometry with orthotic-syntropic application. Right pupil diameter: $4.13 \mathrm{~mm}$ 


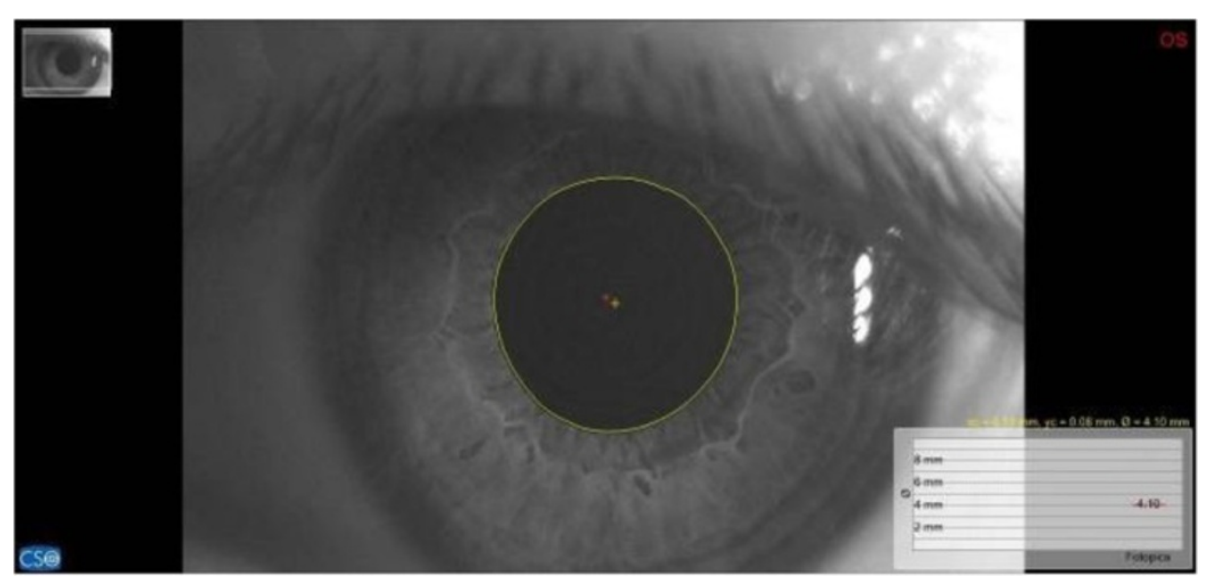

Figure 7 Basal pupillometry with orthotic-syntropic application. Left pupil diameter: $4.10 \mathrm{~mm}$.

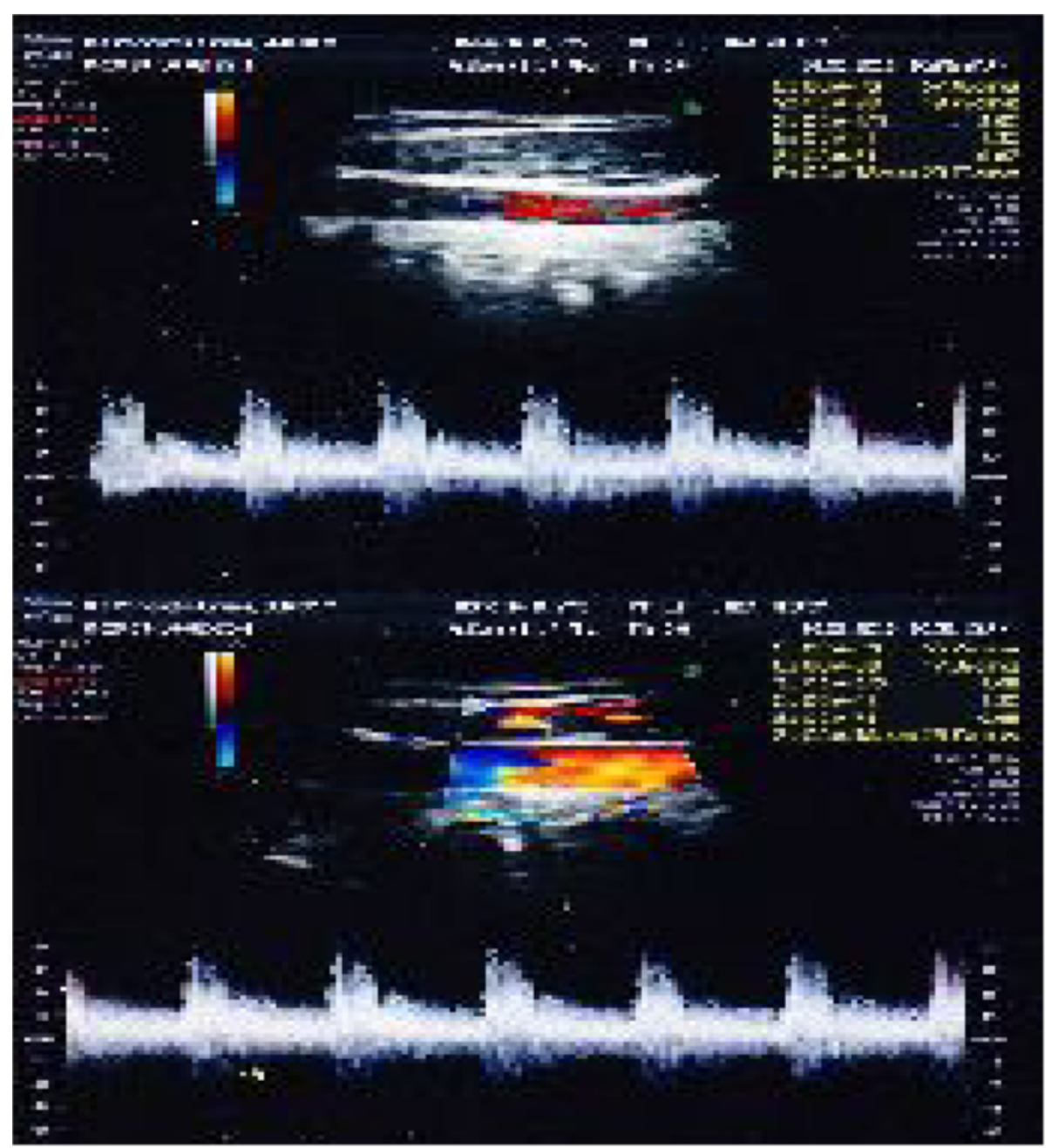

Figure 8 Left (up)/right (down) Carotid duplex color scanner recordings in habitual occlusion. 


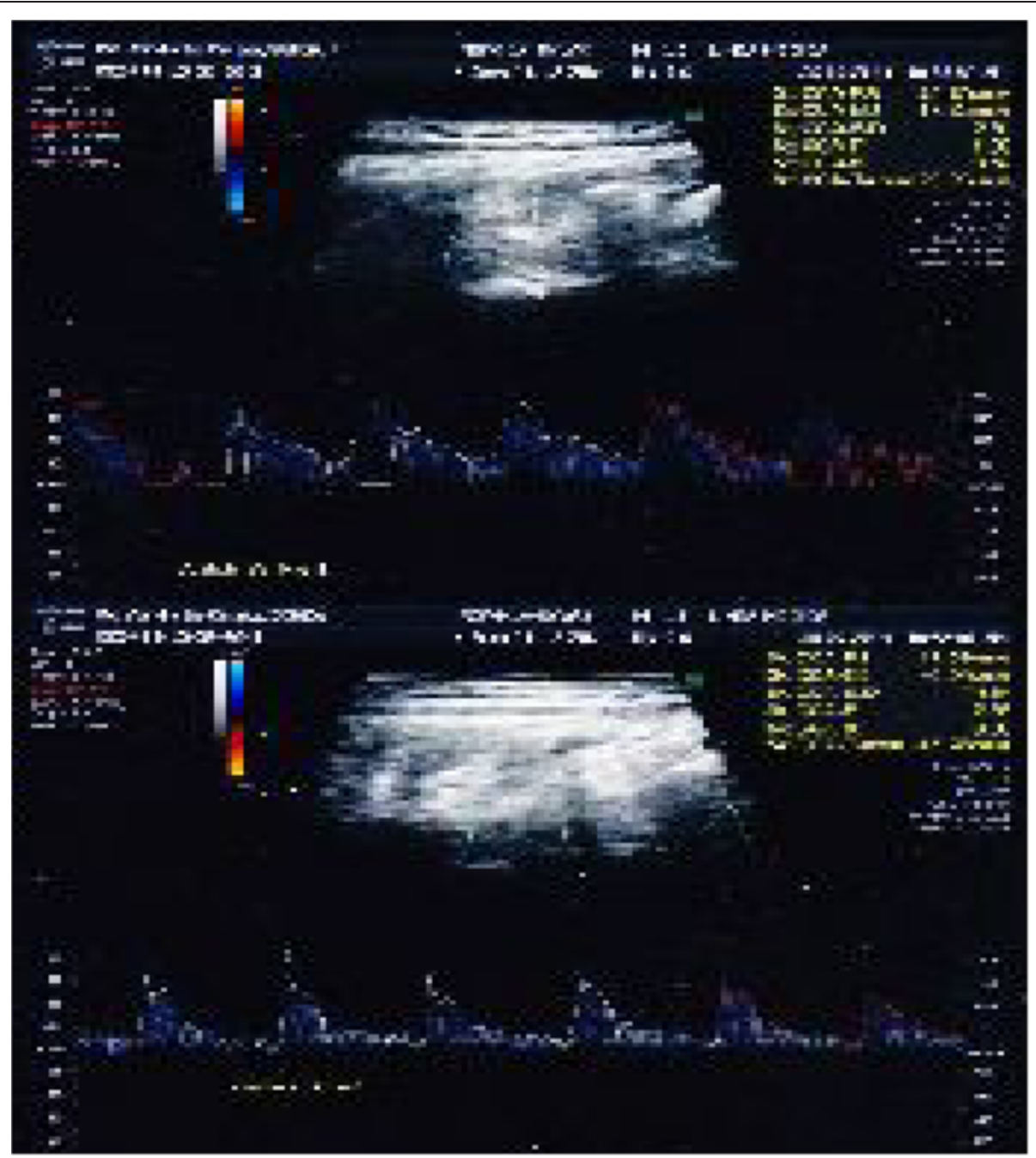

Figure 9 Left (up)/right (down) Vertebral duplex color scanner recordings in habitual occlusion.

results seem to confirm these relationships. In fact, increased right occlusal myoelectric expressivity $(103 \mathrm{mV})$ in our patient is in homolateral relation with her pupillary basal diameter $(4.98 \mathrm{~mm})$ and both exceed the values registered contralaterally, $23 \mathrm{mV}$ and $4.40 \mathrm{~mm}$ respectively. Contrary to expectation, it is her left vertebral artery that presents the most suitable hemodynamic variations and it is more influenced by sympathetic autonomic action. In coherence with what was registered in pupillometric analyses $(4.13 \mathrm{~mm}$ right pupil and $4.10 \mathrm{~mm}$ left pupil), proprioceptive rebalance has determined, even in the cerebro-afferent vascular system, a general reduction of sympathetic autonomic action in almost all hemodynamic evaluations.

The comparative analysis between pupillometric and electromyographic variations is particularly interesting. The complexity of neurophysiological interactions in trigeminal proprioception, which are at the basis of the data registered in this case report, can permit us, at the moment, to hypothesize a different mode of activation of the LC-noradrenaline system. In habitual occlusion, the side with a greater myoelectric expressivity is commonly associated with the preferential side of mastication that determines a greater stimulation of periodontal receptors and increases of muscle splindle discharge frequency. These conditions can increase glutamate release, indirectly for the activation of presynaptic gammaaminobutyric acid-A (GABA- ${ }_{\mathrm{A}}$ ) receptors or directly through the trigeminal mesencephalic nucleus, or in the coerulean and peri-coerulean area [23]. The effects of changes in LC activity on autonomic functions result in complex patterns of neuronal interactions, because the LC exhibits pronounced responses also to non-noxious environmental stimuli $[17,18]$, as in the case of muscle spindle and dental periodontal increases of discharge frequency. Moreover, preliminary findings showed that the LC and trigeminal systems released neuronal vasoactive peptides, as pituitary adenylate cyclase-activating peptide and 


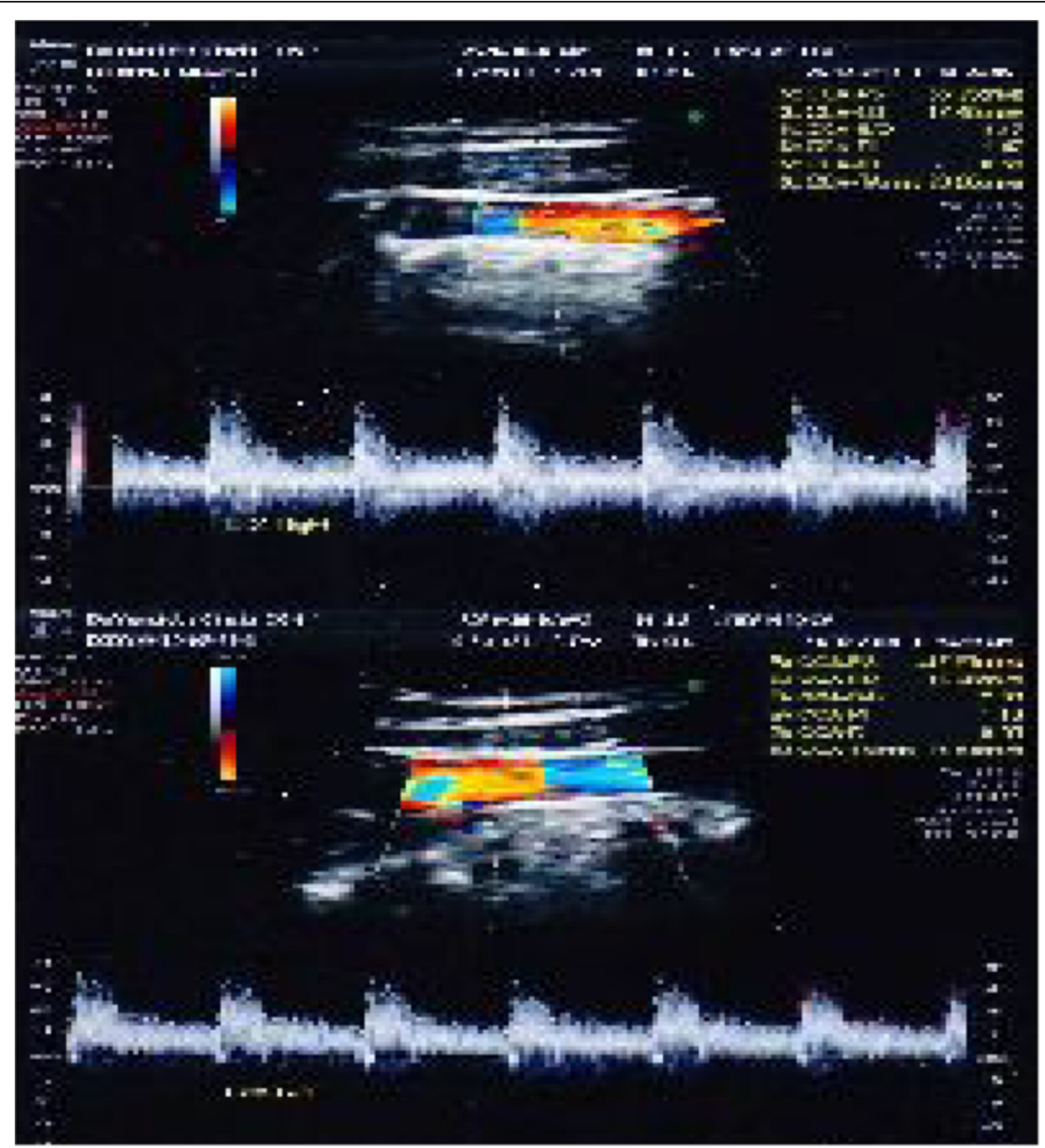

Figure 10 Left (up)/right (down) Carotid duplex color scanner recordings with orthotic-syntropic application.

vasoactive intestinal polypeptide [29,30]. LC and trigeminal asymmetric functions might have determined a concomitant asymmetrical release of these peptides into the autonomic system. In fact, the brainstem organization of the noradrenaline (NA) projections, coming from retrograde as well as anterograde transport studies, showed that the NA inputs to the trigeminal motor nuclei originate almost exclusively in A5 and A7 group cells with almost no contribution from the LC. By contrast, after the deposit of tracer in the rostral part of the spinal trigeminal nucleus, the majority of labeled NA neurons were found in LC [31]. The larger basal pupillometry and the better hemodynamics of the right side should confirm this hypothesis. The symmetrization of the periodontal proprioceptive input and the elimination of the occlusal open bite, both retrieved by the orthotic, may have induced a more appropriate and balanced reduction of the coerulean activity, together with a minor co-release of vasoactive peptides. The $\mathrm{LC}$ contribution to the control of autonomic activity results from direct projections to sympathetic divisions of the spinal cord, including the superior cervical ganglion which becomes innervated in the vertebral artery [15]. This datum could explain the larger and symmetric effects observed both on hemodynamic vertebral and basal pupillometric examinations.

In summary, if recent findings confirmed that the pattern and intensity of muscle contraction during voluntary working side chewing influenced the cerebral blood flow velocity, then it is also possible to affirm that the proprioceptive signals elicited by involuntary occlusion during swallowing can be important. Infact, the frequency of this act, even if for a short time ( 0.7 seconds), is constantly repeated every minute in 24 hours and can modify the hemodynamics of cerebro-afferent vessels as well as that of the sympathetic nervous activity. These effects could be interesting in that chronic cerebral hypoperfusion accelerates amyloid beta-deposition [32]. Hemodynamic parameters indicate a significant vertebral 


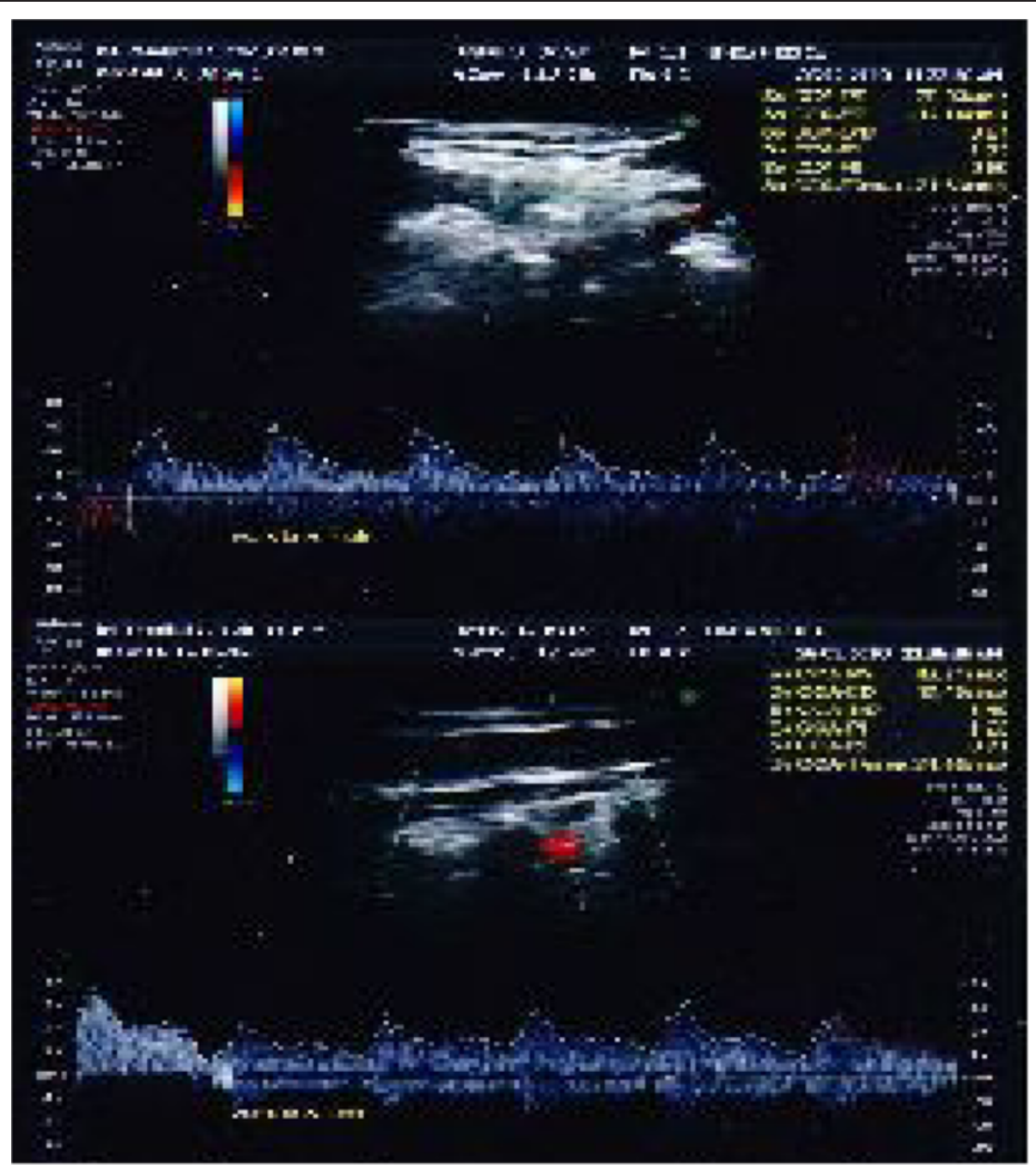

Figure 11 Left (up)/right (down) Vertebral duplex color scanner recordings with orthotic-syntropic application.

Table 1 Hemodynamic variations of cerebro-afferent vessels

\begin{tabular}{|c|c|c|c|c|c|c|c|c|}
\hline \multicolumn{9}{|c|}{ Hemodynamic variations of cerebro-afferent vessels } \\
\hline & \multicolumn{4}{|c|}{ OCCLUSAL UNBALANCE } & \multicolumn{4}{|c|}{ ORTHOTIC APPLICATION } \\
\hline & C.a. & C.a. & V.a. & V.a. & C.a. & C.a. & V.a. & V.a. \\
\hline & Right & Left & Right & Left & Right & Left & Right & Left \\
\hline PI index & 1.30 & 1.30 & 1.0 & 2.88 & 1.42 & 1.16 & 1.23 & 1.23 \\
\hline RI index & 0.67 & 0.68 & 0.62 & 0.99 & 0.68 & 0.65 & 0.69 & 0.71 \\
\hline PS index & 57.40 & 55.27 & 46.45 & 47.58 & 55.35 & 47.97 & 39.03 & 43.21 \\
\hline ED index & 18.79 & 17.87 & 17.82 & 0.64 & 17.48 & 16.96 & 12.16 & 12.70 \\
\hline S-D index & 3.05 & 3.09 & 2.61 & 74.34 & 3.17 & 2.83 & 3.21 & 3.40 \\
\hline
\end{tabular}

systolic pulsatility and average flow velocity: (P.I. Index);

systolic and diastolic relationship-flow: (R.I. Index);

systolic peak in $\mathrm{cm} /$ second: (P.S. Index)

diastasis cordis in $\mathrm{cm} / \mathrm{second}$ : (E.D. Index)

systole-diastole relationship: (S-D Index);

Carotid artery: C.a.

Vertebral artery: V.a. 
artery dysfunction and this could determine, in the long run, microcirculatory variations especially in reticular formation or/and in human cervical cord. These conditions may cause dizziness, drop attack, gait disturbance or cognitive impairment [33].

\section{Conclusions}

Comparative data analysis has shown that an asymmetric involuntary occlusion may represent an interferential pattern on the functional modalities of LC, also involving the sympathetic autonomic systems with effects on pupillometric baseline and cerebral-afferent artery blood flow velocity. Within the limits of this case report, further investigations are necessary in order to detect the modalities, at the moment not completely proved, through which the occlusal proprioception may modulate the widespread LC-noradrenaline neuromodulator projections. As the pupillometric evaluations have proved useful to analyse the LC functional modes, it could be used as a diagnostic tool in the assessment of pathologies involving the LC-noradrenaline system, as cardiological and brain vascular stress-induced diseases. The inclusion of myoelectric masseter evaluations can be useful in patients with asymmetric hemodynamics of cerebro-afferent vessels and the trigeminal proprioceptive rebalance can integrate the complex therapy of patients with increased chronic sympathetic activity. Moreover, the repeated occlusal controls by computerized functional evaluations are essential both for orthodontic and dental prosthesis therapy and to prevent trigeminal proprioceptive unbalance-induced effects.

\section{Consent}

Written informed consent was obtained from the patient for publication of this manuscript and accompanying images. A copy of the written consent is available for review by the Editor-in-Chief of this Journal.

\section{Competing interests}

The author declares that he has no competing interests.

Received: 6 November 2011 Accepted: 7 March 2012

Published: 3 September 2012

\section{References}

1. Momose I, Nishikawa J, Watanabe T, Sasaki Y, Senda M, Kubota K, Sato Y, Funakoski M, Minakuchi S: Effect of mastication on region cerebral blood flow in humans examined by positron-emission tomography with 150-labeled water and magnetic resonance imaging. Arc Oral Biol 1997, 42:57-61.

2. Hasegawa Y, Ono T, Sakagami J, Hori K, Maeda Y, Hamasaki T, Nokubi T: Influence of voluntary control of masticatory side and rhythm on cerebral hemodynamics. Clin Oral Invest 2011, 15:113-118.

3. Lin SK, Chang YI, Ryu SJ, Chu NS: Cerebral hemodynamic responses to betel chewing: a doppler study. Clin Neuropharmacol 2002, 25:244-250.

4. Ono T, Hori K, Ikebe K, Nokubi T, Nago S, Kookaburra I: Factors influencing eating ability of old in-patients in a rehabilitation hospital in Japan. Gerondontology 2003, 20:24-31

5. Bergdahl M, Habib R, Bergdhal J, Nyberg L, Nilsson LG: Natural teeth and cognitive function in humans. Scand J Psychol 2007, 48:557-565.
6. Kato T, Usami T, Noda Y, Hasegawa M, Nabeshima T: The effect of the loss of molar teeth on spatial memory and acetylcholine release from the parietal cortex in aged rats. Behav Brain Res 1997, 83:239-242.

7. Watanabe K, Onozuka M, Fujita M, Ozono S: Changes in the septohippocampal cholinergic system following removal of molar teeth in the aged SAMP8 mouse. Behav Brain Res 2002, 133:197-204.

8. Kaoruko Y, Wakabaiashi N, Kobayashi T, Suzuki T: Effect of tooth loss on spatial memory and trkB-mRNA levels in rats. Hippocampus 2008, 18:542-547.

9. Sugiyama K, Okumura C, Watanabe S: Validation of transcranial Doppler method to evacuate the effects of mastication on cerebral blood flow. Japanese J Nursing Res 1999, 32:473-482.

10. Nakamura Y, Katakura N: Generation of masticatory rhythm in the brainstem. Neurosci Res 1995, 23:1-9.

11. Notsu K, Tumori T, Yokota S, Semine J, Yasui Y: Posterior lateral hypothalamic axon terminal are in contact with trigeminal premotor neurons in the parvicellular reticular formation of rat medulla oblongata. Brain Res 2008, 1244:71-81

12. Van Bockstaele EJ, Aston-Jones G: Integration in the ventral medulla and coordination of sympathetic pain and arousal functions. Clin Exp Hypertens 1995, 17:153-165.

13. Rajkoski J, Kubiak P, Aston-Jones G: Correlations between locus coeruleus (LC) neural activity, pupil diameter and behaviour in monkey support a role of LC in attention. Soc for Neurosc Abstracts 1993, 19:974.

14. Elam M, Svensson $T H$, Thoren P: Locus coeruleus neurons and sympathetic nerves: activation by cutaneous sensory afferents. Brain Res 1986, 366:254-261.

15. Samuels ER, Szabadi E: Functional neuroanatomy of the noradrenergic locus coeruleus: its roles in the regulation of arousal and autonomic function part I: principles of functional organisation. Current Neuropharmacology 2008, 6:235-253.

16. Cedarbaum JM, Aghajanian GK: Afferent projections to the rat locus coeruleus as determined by a retrograde tracing technique. J Comp Neurol 1978, 178(1):1-16.

17. Aston-Jones G, Bloom FE: Norepinephrine-containing locus coeruleus in behaving rats exhibit pronounced responses to non-noxious environmental stimuli. J Neurosci 1981, 1:887-900.

18. Sara S, Herve A: Plasticity of auditory responses of locus coeruleus neurons: studies in anesthetized rats. Soc Neurosci Abstr 1993, 19:413.

19. Luo P, Zhang J, Yang R, Pendlebury W: Neuronal circuitry and synaptic organization of trigeminal proprioceptive afferents mediating tongue movement and jaw-tongue coordination via hypoglossal premotor neurons. European J Neuroscience 2006, 23:3269-3283.

20. Couto LB, Moroni CR, dos Reis Ferreira CM, Elias-Filho DH, Parada CA, Pela IR, Coimbra NC: Descriptive and functional neuroanatomy of locus coeruleus-noradrenalin-containing neurons involvement in bradykinininduced antinociception on principal sensory trigeminal nucleus. J Chem Neuroanat 2006, 32(1):28-45.

21. Panneton WM, McCulloch PF, Sun W: Trigemino-autonomic connections in the muskrat: the neural substrate for the diving response. Brain Res 2000, 874(1):48-65.

22. Yabushita T, Zeredo JL, Toda K, Soma K: Role of occlusal vertical dimension in splindle function. J Dent Res 2005, 84(3):245-249.

23. Koga H, Ishibashi H, Shimada H, II-Sung J, Nakamura TY, Nabekura J: Activation of presynaptic GABA-A receptors increase spontaneous glutamate release onto noradrenergic neurons of the rat locus coeruleus. Brain Res 2005, 1046:24-31.

24. Nieuwenhuys $R$, Geeraedts $L M$, Veening JG: The medial forebrain bundle of the rat. I. General introduction. J Comp Neurol 1982, 206(1):49-81.

25. Batini C, Rossi GF, Zanchetti A, Moruzzi G: Brain stem reticular formation. Anatomy and Physiology. Arch Ital Biol 1959, 95:199-435.

26. van der Kooy D, Phillips AG: Involvement of the trigeminal motor system in brain stem self- stimulation and stimulation-induced behavior. Brain Behav Evol 1979, 16:293-314.

27. Takamatsu J, Inoue T, Tsuruoka M, Suganuma T, Furuya R, Kawawa T: Involvement of reticular neurons located dorsal to the facial nucleus in activation of the jaw-closing muscle in rats. Brain Res 2005, 1055(2):93-102.

28. Didier H, Marchetti C, Borromeo G, Tullo V, Bussone G, Santoro F: Persistent idiopathic facial pain: multidisciplinary approach and assumption of comorbidity. Neurol Sci Suppl 2010, 1:s189-s195. 
29. Baeres FM, Møller M: Origin of PACAP-immunoreactive nerve fibers innervating the subarachnoidal blood vessels of the rat brain. $J$ Cereb Blood Flow Metab 2004, 24(6):628-635

30. Goadsby PJ, MacDonald GJ: The effect of infusion of various peptide antisera on vasodilatation in the cat common carotid vascular territory. Clin Exp Neurol 1985, 21:115-121.

31. Lyons WE, Grzanna R: Noradrenergic neurons with divergent projections to the motor trigeminal nucleus and the spinal cord: a double retrograde neuron labeling study. Neuroscience 1988, 26(2):681-693.

32. Kitaguchi $H$, Tomimoto $H$, Ihara M, Shibata M, Uemura K, Kalaria RN, Kihara T, Asada-Utsuqi M, Kinoshita A, Takahashi R: Chronic cerebral hypoperfusion accelerates amyloid beta-deposition in APPSwlnd transgenic mice. Brain Res 2009, 1294:202-210.

33. Niedermeyer E: Vertebrobasilar artery insufficiency and electroencephalogram. Clin EEG Neurosci 2008, 39(1):8-11.

doi:10.1186/1752-1947-6-275

Cite this article as: De Cicco: Cerebro-afferent vessel and pupillary basal diameter variation induced by stomatognathic trigeminal

proprioception: a case report. Journal of Medical Case Reports 2012 6:275.

\section{Submit your next manuscript to BioMed Central and take full advantage of:}

- Convenient online submission

- Thorough peer review

- No space constraints or color figure charges

- Immediate publication on acceptance

- Inclusion in PubMed, CAS, Scopus and Google Scholar

- Research which is freely available for redistribution 